\title{
As práticas desenvolvidas no Programa Saúde da Família contribuem para transformar o modelo de atenção à saúde?
}

Do the practices developed in Family Health Program contribute to transform the present model of health care? ¿Las competencias desarrolladas en el Programa de Salud de la Familia logran la transformación del modelo de atención a la salud?

\author{
Helena Eri Shimizu', Carlos Rosales" \\ 'Universidade de Brasília. Departamento de Enfermagem. Brasília, DF \\ "Organização Panamericana de Saúde. Whashington, DC
}

Submissão: $28 / 06 / 2008$

Aprovação: 24/03/2009

\section{RESUMO}

O estudo tem como objetivos identificar e analisar as principais práticas desenvolvidas no Programa Saúde da Família. Trata-se de um estudo de caso da Unidade do Programa Família Saudável de São Sebastião, DF, cujos dados foram obtidos por meio de entrevista semi-estruturada com trabalhadores de uma eQuipe básica e observação do processo de trabalho. Conclui-se Que são desenvolvidas diversas práticas fundamentais, mas outras voltadas para a promoção da saúde da comunidade são necessárias para o alcance do modelo de atenção.

Descritores: Atenção básica; Programa Saúde da Família; Prática profissional.

\begin{abstract}
This study aimed to identify and analyze the main primary health care practices developed in the Family Health Care Program. Qualitative case study was carried out in the region of São Sebastião, DF. Data were collected through semi-structured interviews with team workers and observation of the work process. The author concluded that diverse basic practices are developed in primary health care, but others practices focused in health care promotion are necessary in order to transform the health care model.
\end{abstract}

Descriptors: Primary Health Care; Family Health Program; Professional Practice.

\section{RESUMEN}

El estudio tiene por objetivo identificar y analizar las principales prácticas desarrolladas en la atención primaria. Se trata de un estudio de caso de carácter cualitativo centrado en la Unidad del Programa de la salud de la Familia del municipio de Sebastião, DF. Los datos se obtuvieron por medio de una entrevista semi-estructurada con los trabajadores de un equipo básico, así como através de la observación del proceso de trabajo. El estudio concluye Que, si bien se desarrollan diversas prácticas, otras prácticas orientadas hacia la promoción en la salud se hacen necesarias para el alcance del modelo de atención.

Descriptores: Atención Primaria de Salud; Programa Salud de la Familia; Práctica Profesional 


\section{INTRODUÇÃO}

A melhoria do sistema de saúde requer investimento na Atenção Primária (APS), porque se constitui pilar para um sistema de saúde Que proporcione atenção à saúde de Qualidade, resolutiva e de baixo custo $^{(1-3)}$. É nessa perspectiva Que a Organização PanAmericana de Saúde (OPAS), ao reconhecer as iniQüidades em saúde cada vez mais amplas, os sistemas de saúde permanentemente sobrecarregados e Que colocam em risco a saúde da população, tem desenvolvido diversas estratégias para a renovação da APS ${ }^{(4)}$.

No Brasil, com a implantação do Sistema Único de Saúde (SUS) Que tem como princípios: universalidade, integralidade e equidade, começou-se a investir mais profundamente na Atenção Primária Saúde, denominada Atenção Básica $(\mathrm{AB})$ e definida como um conjunto de ações de caráter individual e coletivo voltadas para a prevenção dos agravos, o tratamento e a reabilitação e manutenção da vida ${ }^{(5)}$, e Que tem como os seus elementos essenciais: o foco na família, universalidade, acessibilidade, coordenação do cuidado, do vínculo e continuidade, integralidade, orientação para a comunidade, capacitação profissional.

A Estratégia Saúde da Família (ESF) é considerada prioritária para transformação do modelo de atenção, a partir da reorganização da $A B$, na Qual é proposta uma nova forma de cuidar da saúde: para populações delimitadas em territórios adstritos, com ênfase na atenção a família, no trabalho em equipe multiprofissional, no planejamento local participativo e na parceria com outras instituições e outros setores, nas ações de prevenção, promoção, cura e reabilitação ${ }^{(5-6)}$.

Sabe-se, contudo, Que o processo de mudança no modelo de atenção, entendido como "combinações de saberes (conhecimentos) e técnicas (métodos e instrumentos) utilizados para resolver problemas e atender as necessidades de saúde individuais e coletivas $^{(7-8)}$ é extremamente complexo e gradual. Exige alterações macro-sistêmicas, Que dizem respeito a mudanças na formulação e implantação de políticas e Que proporcionem condições para mudanças em nível micro, especificamente no cotidiano do fazer saúde nos serviços de $A B$, Que tem como um dos desafios operar mudanças no processo de trabalho, o Que requer a redefinição do objeto, meios e instrumentos e finalidade. Isso demanda análise dos saberes e tecnologias, bem como das relações estabelecidas entre os sujeitos, profissionais e trabalhadores da saúde e a população usuária do sistema ${ }^{(8)}$.

Certamente o novo modelo de cuidado proposto exige da equipe de trabalho a aQuisição de novas competências, entendida como a mobilização de um saber em ato, construída na relação com o outro, ou seja, no processo do cotidiano de trabalho junto à população ${ }^{(9-1)}$. É necessária a utilização da abordagem dialógica de competências, Que permite entender Que as práticas profissionais são compostas por diferentes combinações, Que respondem satisfatoriamente aos padrões estabelecidos, permitindo Que os trabalhadores desenvolvam um estilo singular, adequado e eficaz ${ }^{(9,10)}$. Nessa perspectiva, o processo de trabalho deve ser visto como uma série de eventos relacionando-se uns com os outros, num regime de constante modificação da forma de produzir ${ }^{(1)}$.

Atualmente, a OPAS, para desenvolvimento das equipes da AB, tem investido na definição de competências laborais ${ }^{(4)}$. São consideradas competências gerais: a comunicação, Que se constitui ferramenta essencial para o trabalho em equipe, interação com o usuário, comunidade, e outros serviços e setores; a análise das informações do sistema de saúde, para avaliação do estado de saúde da população, das atividades de promoção e prevenção e curativas desenvolvidas; a gestão de recursos humanos para o desempenho do trabalho em equipe, inclusive diagnosticando as dificuldades do trabalho desenvolvido na unidade e com outros níveis de atenção à saúde e o conhecimento das funções essenciais de saúde pública (FESP) ${ }^{(4,12)}$.

As FESP são definidas como "um conjunto de atividades fundamentais e imprescindíveis para proteger a saúde da população e tratar a enfermidade por meios dirigidos ao ambiente e a

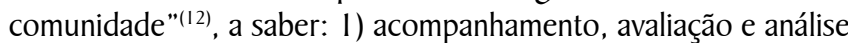
dos problemas de saúde, 2) vigilância em saúde pública, investigação e controle de riscos e danos de saúde pública, 3) promoção da saúde, 4) participação dos cidadãos em saúde, 5) desenvolvimento de políticas e capacidade institucional para planejamento e gestão em saúde pública; 6) fortalecimento da capacidade institucional de regulação e fiscalização da saúde pública, 7) avaliação e promoção do acesso eqüitativo a serviços de saúde necessários; 8) desenvolvimento de recursos humanos e capacitação em saúde pública; 9) garantia e melhoria da Qualidade dos serviços de saúde individuais e coletivos, 10) investigação em saúde pública; I1) redução de impacto das emergências e desastres em saúde pública.

Acredita-se Que, se estas funções estão bem desenvolvidas pelos serviços de $A B$, ficará assegurado o trabalho de saúde pública ${ }^{(4,11,12)}$ e, conseqüentemente, haverá maior possibilidade de alcance do modelo de atenção requerido. Assim, a análise das práticas, entendidas como o conjunto de ações desenvolvidas no cotidiano, articulado em operações, Que impõe uma estratégia de ação sobre os determinantes e condicionantes dos problemas ou sobre os efeitos da existência deles em um território determinado, é de suma importância verificar se ocorrem transformações no modelo de atenção à saúde centrada na Qualidade de vida das pessoas e do seu ambiente, bem como na relação humanizada entre equipe de saúde e comunidade, especialmente com as famílias.

São objetivos do estudo: analisar as práticas desenvolvidas no processo de trabalho na Atenção Básica - Programa Família Saudável (PFS) a fim de verificar as suas potencialidades e limitações para o alcance da mudança do modelo de atenção à saúde.

\section{METODOLOGIA}

Trata-se de um estudo com método Qualitativo. Foi escolhida para este estudo, uma das unidades da Regional Administrativa 16 do Distrito Federal, a de São Sebastião, Que atendia os critérios de inclusão: ser uma unidade urbana, com o funcionamento exclusivo do Programa Família Saudável (PFS) há pelo menos seis meses, com a equipe mínima completa (médico, enfermeira, auxiliares de enfermagem e agentes de saúde) e obtenção do aceite da Coordenação Regional de Saúde para participar da pesquisa.

Foi realizada observação do processo de trabalho realizado pelos seguintes agentes: médico, enfermeira, auxiliares de enfermagem e agentes comunitários de saúde (ACS) com registros em diário de campo $^{(13)}$. Utilizou-se um roteiro para subsidiar as observações de campo, a saber: as ações e modo como são desenvolvidas pelos agentes, a interação e a articulação entre os agentes, a relação dos 
agentes com os usuários. A saturação dos dados, ou seja, a repetição das ações e interações desenvolvida por todos os trabalhadores determinou a Quantidade de tempo da observação, duas semanas, Que totalizou 80 horas.

Realizaram-se também entrevistas semi-estruturadas com um médico, uma enfermeira, dois auxiliares de enfermagem e Quatro agentes comunitários de saúde, com gravação em áudio. O roteiro utilizado nas entrevistas continha as seguintes Questões: Quais são as atividades desenvolvidas? Qual a relação do trabalho Que desenvolvem com o trabalho dos demais agentes? Quais são as formas de acompanhamento, controle e avaliação das ações, e as percepções sobre o PFS.

O projeto de pesquisa foi aprovado pelo Comitê de Ética da Secretaria Estadual de Saúde do DF (SES-DF), os sujeitos do estudo foram informados sobre os objetivos e procedimentos da pesQuisa, da garantia do sigilo da identidade mediante designação de código individual e assinaram o Termo de Consentimento Livre e Esclarecido.

Os dados obtidos foram analisados seguindo-se alguns passos da análise de conteúdo propostos por Minayo ${ }^{(13)}$, a saber: 1) ordenação dos dados, Que englobou a transcrição na íntegra das entrevistas, 2) leitura exaustiva e posterior organização dos depoimentos e dos dados obtidos na observação e 3) finalmente a classificação dos dados, Que deu origem aos temas abaixo apresentados.

\section{RESULTADOS E DISCUSSÃO}

As práticas relativas a diagnóstico, monitoramento e avaliação das ações necessárias à saúde da comunidade

Verificou-se Que o ACS tem contribuição relevante no desempenho da "identificação da situação de saúde da população", pois visita cerca de dez famílias diariamente, onde aborda de forma pontual a existência de problemas de saúde, como mostra o depoimento:

...minha função é ir na casa do paciente, levar receita, levar remédio e visitar de preferência os acamados, gestantes, idosos, crianças de baixo peso... (ACS I).

As famílias são muito receptivas e costumam apresentar os seus problemas, demonstrando confiança e aceitação do ACS. Essa aproximação, possivelmente é favorecida pelo conhecimento do modo de vida da comunidade pelo ACS, por ele ser também morador da região.

Há uma reunião breve, esporadicamente, em Que o ACS compartilha com os demais elementos da equipe as informações sobre os problemas de saúde identificados nas famílias e discute as decisões para solucioná-los. Contudo, as Questões mais urgentes e freqüentes demandadas pelas famílias são resolvidas pelo ACS diretamente com médico ou com a enfermeira. Os demais profissionais, usualmente, visitam apenas os pacientes Que requerem maior complexidade de cuidados ou encontram-se impossibilitados fisicamente.

Foi observado Que a equipe toma como objeto de trabalho o cuidado à família e tem buscado responder por meio das visitas as suas necessidades, mas as ações desenvolvidas demonstram Que estão focados em problemas pontuais e fundamentam-se, basicamente, nos conhecimentos da epidemiologia e fisiopatologia, Que limita sobremaneira a compreensão do complexo fenômeno processo saúde-doença, revelando a necessidade de rever a forma de abordagem das famílias em domicílio. O aprimoramento das competências relativas a abordagem familiar requer, primeiramente, a discussão sobre o Que se entende por família para facilitar o planejamento, a execução das ações e avaliação. A multiplicidade de abordagens de família utilizada por uma equipe Que não dialoga entre si ou não se complementa cria um Quadro de diversidades e parcialidades Que não permite o alcance da integralidade no cuidado da família ${ }^{(14)}$.

Ressalta-se Que se constatou Que o sistema de informação em saúde local é precário, pois os dados obtidos junto às famílias, por ocasião do cadastramento, os atendimentos realizados, as atividades desenvolvidas pela equipe, dentre outros, não são adeQuadamente valorizados pela equipe, ou seja, cumprem apenas uma função burocrática de gerar estatísticas sobre as atividades realizadas, o Que resulta na ausência de diagnóstico das necessidades de saúde, de planejamento sistematizado e de avaliação das ações para a população na área de abrangência da equipe.

Como conseqüência da falta de um planejamento sistematizado, o serviço se organiza para realizar o atendimento à comunidade segundo um cronograma semanal Que orientam as ações de todos os elementos da equipe e é composto das seguintes ações: consultas de Clínica Médica, consultas de Controle de Crescimento e Desenvolvimento (crianças de 0-5 anos) e Pré-Natal, coleta de material para prevenção de Câncer de Colo Uterino, atendimento a grupos e visitas domiciliares. Eventualmente são realizadas outras atividades.

Houve nosso primeiro passo, nós planejarmos em equipe, fizemos o nosso cronograma de trabalho, então eu anunciei o cronograma. Para Quê? Para Que nós soubéssemos que dia eu estaria fazendo visita domiciliar, Que dia eu estaria fazendo consultas a gestante, Que dia eu estaria fazendo a prevenção ginecológica, fazendo consultas de verminose. (Enf I).

Estes dados indicam Que é importante fortalecer a competência da equipe do PFS para organização e análise do sistema de informação da $A B$, Que reQuerem o envolvimento de todos os membros da equipe, bem como da comunidade, para a realização de planejamento, monitoramento e avaliação Que atendam as amplas necessidades da população.

\section{Práticas relativas à atenção a indivíduos}

Observou-se que grande parte da agenda da equipe do PFS é reservada para resolução de problemas da demanda espontânea, clínico individual e de pequena complexidade. As consultas de Clínica Médica são agendadas previamente. Contudo, diariamente, há um excedente de pessoas com problemas de saúde, Que passam por uma triagem feita pelo auxiliar de enfermagem e os mais urgentes atendidos no mesmo dia. Os demais têm a consulta agendada para outra data.

São realizadas consultas às crianças de $0-5$ anos do Programa Crescimento e Desenvolvimento (CD) e das gestantes do Programa de Pré-natal. Além disso, a enfermeira faz a coleta de material para prevenção de Câncer de Útero. 
Constatou-se Que, cotidianamente, todos profissionais se empenham para Que as consultas sejam realizadas rapidamente. É da competência do auxiliar de enfermagem realizar a pré-consulta, Que consiste na tomada de algumas informações e dos sinais vitais e do ACS é organizar os prontuários dos pacientes. A percepção da equipe é de Que a atenção despendida no processo da triagem é uma forma de garantir a humanização no atendimento. Todavia, constatou-se Que tem como finalidade precípua diminuir o tempo das consultas, focadas basicamente nas Queixas dos pacientes e solucionadas com a prescrição de medicamentos.

Os dados acima apresentados demonstraram Que a equipe desenvolve diversas ações embasadas no modelo clínico tradicional, cuja finalidade principal é atender à pressão das demandas imediatas de baixa complexidade e de alguns segmentos da população considerados mais vulneráveis. Trata-se de uma prática clínica empobrecida Que consiste na realização de ações curativas superficiais e limitadas $^{(15)}$. Caso essa forma de organização das ações se cristalize, corre-se o risco do PFS tornar-se linha auxiliar do modelo hegemônico.

Outro aspecto verificado é Que a população tem problema para realizar Qualquer tipo de exame, devido escassez de recursos em condições adequadas em todos os serviços disponibilizados. No depoimento abaixo se verifica Que os profissionais sentem-se constrangidos por não garantirem atendimento ao usuário dos serviços de referência de outros níveis de atenção.

...Então eles ficam frustrados que os encaminhamentos da gente, por exemplo, eu encaminho muito mamografia, e tem muita gente Que eu detecto que está com nódulo de mama eu encaminho pra mamografia, chega lá, não é atendido porQue o HRAN ta com a máQuina Quebrada, o Hospital de Base tá com a máquina Quebrada, e o HUB está superlotado porque os parceiros da Secretaria de Saúde foram pra lá, né, fizeram parceria e foram pra lá. Aí isso gera, deixa eles: pra que que eu vou procurar (Enf I).

O PFS não está cumprindo satisfatoriamente a sua função de porta de entrada do sistema de saúde, Que tem como objetivo tornar o clínico geral o coordenador da assistência aos usuários pela rede assistencial na busca de atenção necessária e de QualQuer nível assistencial ${ }^{(3,16,17)}$. É necessária a construção de estratégias e articulações que permitam o acesso ao serviço de saúde, entendido como a possibilidade de entrada nos serviços requeridos, o recebimento da atenção por profissionais Qualificados e utilização de recurso tecnológico adequado às necessidades dos pacientes. Além disso, há Que ser alcançada a longitudinalidade, entendida como um fenômeno Que envolve a disponibilidade regular de assistência e decisão do usuário para buscá-la a Qualquer tempo Que necessitar. Pressupõe também Que a comunicação entre profissional e paciente ocorra de forma satisfatória, pautado na possibilidade de formação de vínculo ${ }^{(1)}$.

\section{Práticas relativas à atenção a coletivos}

Das práticas voltadas à Atenção a Grupos, verificou-se Que funcionam dois grupos aos pacientes com Diabetes e com Hipertensão, onde são desenvolvidas consultas Que são competências específicas do médico e da enfermeira e outras atividades Que visam o controle e prevenção da doença. A equipe demonstrou Que é necessário envidar esforços para a manutenção dos usuários nos grupos, pois eles não têm o hábito de controlar e promover sistematicamente a sua saúde, como o depoimento abaixo.

Ninguém Quer se prevenir. As vezes pode chegar e prevenir uma gripe, sei lá. Você pode prevenir outra doença, mas você não previne, você espera acontecer e aí vem para o PSF e Quer ser atendido (ACS2).

Nos intervalos das consultas às crianças e gestantes são realizadas palestras educativas, assumidas freeüentemente pelo auxiliar de enfermagem, mas Que contam com a presença de outros profissionais, Que ajudam esclarecer e/ ou aprofundar os conteúdos tratados. São tratados temas relativos a cuidados com a higiene, alimentação e alguns cuidados específicos. Eventualmente são feitas, também, palestras nas escolas e na comunidade sobre assuntos diversos, e Que se observa ser de competência mais direta da enfermeira e do ACS. Constatou-se o predomínio da prática pedagógica tradicional, com utilização de recursos pedagógicos precários e linguagem acessível, mas pouco interativa e Que culmina na pouca participação dos usuários. É evidente a necessidade de adoção de estratégias comunicacionais dialógicas, Que permitam conhecer o indivíduo, incluindo as suas crenças, valores e as condições objetivas em Que vivem. Essa forma de abordagem parte do suposto Que o usuário é portador de um saber, diverso do saber técnico, mas que deve ser valorizado, a fim de capacitá-los a decidirem sobre as estratégias para promover, manter e recuperar a sua saúde.

Ocasionalmente são feitos mutirões para vacinação, destinados às crianças até cinco, anos para administração do reforço da vacina contra paralisia infantil. Nessa oportunidade são também captadas crianças Que não receberam outros tipos de vacinas e eventualmente, se permite aos adultos acompanhantes para tomarem as vacinas disponíveis (anti-têtanica e gripe para os idosos). São realizados, também, outros mutirões em saúde Que visam a prevenção de doenças como Câncer de Próstata, Câncer de Pele, entre outros. Observou-se participação satisfatória da comunidade Que, normalmente, não tem oportunidade para fazer este tipo de avaliação de saúde.

Pôde-se constatar Que a equipe toda demonstrou competências para atender a uma situação de emergência em saúde pública Quando houve o aparecimento de Hantavirose, doença causada por ratos silvestres e Que surgiram em uma cidade satélite em função do desequilíbrio ecológico devido crescimento urbano desordenado. Como se tratava de uma doença pouco conhecida e que havia gerado algumas mortes, a população da cidade e municípios vizinhos demonstrou sentimentos de pânico. A equipe demonstrou ter tido capacidades para desenvolver um plano de ações para esclarecimento da população sobre as formas de prevenção e controle da doença nas escolas e na comunidade.

Os dados relativos às práticas desenvolvidas à coletividade demonstram Que as equipes do PFS utilizam o modelo de ações programáticas. Entretanto, cabe crítica a forma de operacionalização dessas ações, tanto em nível individual como coletivo, pois seria necessária a sua "refuncionalização", ou seja, tomá-las como instrumento de redefinição do processo de trabalho, a partir da identificação dos problemas e necessidades dos indivíduos e grupos 
da população em situações concretas ${ }^{(8)}$.

Ademais, é fundamental percorrer a garantida à integralidade da atenção, considerada um dos pilares fundamentais do Sistema Único de Saúde (SUS), pois este preconiza a priorização das ações de promoção, garantia da atenção nos três níveis de complexidade, articulação das ações prevenção, promoção e recuperação; abordagem integral dos indivíduos e das famílias ${ }^{(2,3,5,16,17)}$.

Práticas relativas a busca de construção de parceria entre equipe de saúde e comunidade na organização dos serviços de saúde

Os dados demonstraram Que, embora a equipe tenha se empenhado para Que a comunidade valorize o PFS, a sua credibilidade é muito frágil, causada pela sucessiva interrupção do programa a cada mudança de governo, e Que gera modificações importantes, especialmente pela mudança constante da equipe impedir a criação de vínculo entre profissionais e usuários.

A comunidade reage de maneira negativa, porque ela vê que o programa não serve. Então esse aspecto, é exatamente esse aspecto, que o programa não é efetivo, o programa não é constante, ele não é verdadeiro, ele aparece e desaparece, aparece e desaparece ...É da incompatibilidade, é da inconstância (MI).

Em relação a finalidade do PFS para a comunidade, verifica-se Que na percepção da equipe houve melhoria do acesso aos serviços básicos de saúde, e principalmente maior aproximação dos moradores da região, Que tem possibilitado a criação de maior vínculo.

...minha visão é Que o PFS veio para descongestionar a saúde em si, e para nós de São Sebastião, descongestionar o posto de saúde e pra trazer melhor atendimento pra comunidade, pra ter mais essa ligação, próxima da comunidade com a saúde, Que hoje a gente tem acesso a médico, enfermeiro, coisas que há tempos atrás agente não tinha, né? Era muito formal o convívio de comunidade e saúde. E hoje está mais, está mais assim, familiarizado, está mais comum e mais fácil, né?(ACS3).

Entretanto, percebe-se Que seria necessária a construção de parceria efetiva entre profissionais e os usuários para identificação das necessidades local de saúde mais amplas e fortalecimento da participação social. Todavia, observou-se Que o modo de organização no trabalho, Que diz respeito a hierarQuia e divisão de trabalho, pauta-se no modelo clássico de gestão, onde cada trabalhador desenvolve isoladamente as suas competências específicas desarticuladas do processo de trabalho como um todo, dificultando a incorporação do usuário. Seria necessário o envolvimento do usuário como protagonista do seu modo de viver e produzir o cuidado em saúde ${ }^{(18)}$.

Nesse sentido, percebe-se Que os treinamentos e capacitações em serviço poderiam contribuir para aQuisição das competências relativas a gestão compartilha pela equipe e usuários. Observouse, porém, Que os treinamentos são precários voltados apenas para a melhoria das competências técnicas e humanísticas. A incorporação da educação permanente seria fundamental para ampliar os momentos para a equipe refletir sobre formas de construção de um projeto assistencial para a comunidade Que inclua a sua participação e a de outros setores da sociedade Que possam contribuir com a melhoria do modo de viver ${ }^{(18)}$.

Há que se destacar, o conselho local de saúde representa um espaço importante de interlocução gestor, usuário e profissional, pois têm a atribuição de acompanhar o planejamento, monitoramento e avaliação das ações de saúde. Nesse sentido, é importante enfatizar Que a participação social em saúde encontrase em plena construção com múltiplas possibilidades, não se pode também prescindir, portanto, da integração e articulação da comunidade com outros setores, como educação, saneamento, assistência social, entre outros, para o fortalecimento das ações em saúde.

Evidencia-se que a construção destas parcerias e a emancipação dos sujeitos envolvidos na participação social em saúde são importantes elementos para construção das ações intersetoriais, fundamentais para a busca da concretização da concepção ampliada de saúde.

\section{CONSIDERAÇÕES FINAIS}

Os resultados do estudo demonstram Que a saúde dos indivíduos e dos grupos é tomada como objeto de trabalho, mas a família ainda é tratada seguindo-se o modelo biológico, o Que tem culminando no desenvolvimento de uma atenção focal e fragmentada, revelando a necessidade de ampliar a sua compreensão, a partir de um diagnóstico mais amplo de suas necessidades físicas, psicológicas, sociais, econômicas e culturais.

Nos meios e instrumentos utilizados no processo de trabalho verificam-se o desempenho de práticas voltadas para o diagnóstico das necessidades de saúde da comunidade, o atendimento clínico individual de baixa complexidade da demanda espontânea e as ações de prevenção, promoção para indivíduos e grupos. Percebeu-se, contudo, Que essas ações não têm como base o diagnóstico sistematizado de suas necessidades, o Que revela urgência da equipe adQuirir competências relativas a construção de planos de saúde local a partir da analise da situação de saúde, com a participação conjunta de gestores, profissionais e usuários.

Em relação a finalidade do PSF, depreendeu-se Que as práticas desenvolvidas pela equipe do PFS têm contribuído para melhoria do acesso da comunidade aos serviços básicos de saúde. Além disso, tem modificado lentamente o modelo de atenção na $A B$, principalmente aproximando o serviço de saúde da população e introduzindo noções de prevenção e promoção da saúde da família. Percebe-se ser imprescindível tomar como objeto os problemas de saúde e seus determinantes, organizando a atenção de forma a incluir não apenas as doenças e suas complicações, mas as ações Que incidam sobre as causas, como condições de vida, trabalho e lazer.

Evidencia-se a necessidade de melhorar as práticas relativas à gestão do serviço por todos os atores envolvidos no processo, gestor, profissionais e usuários, e Que consiste na habilidade de avaliar os problemas encontrados e prover os recursos necessários, a fim de garantir a integralidade da atenção, Que reQuer a integração dos serviços por meio de redes assistenciais, respeitando-se a interdependência entre atores e organizações, uma vez Que nenhuma delas possui a totalidade dos recursos necessários para a resolução dos problemas de uma população em seu ciclo de vida. 


\section{REFERÊNCIAS}

1. Starfield B. Atenção primária: equilíbrio entre necessidades de saúde, serviços e tecnologia. Brasília: Unesco; 2002.

2. Mendes EV. A atenção primária à saúde no SUS. Fortaleza: Escola de Saúde Pública do Ceará; 2002.

3. Giovanella L, Sarah E, Mendonça MH. Porta de entrada pela atenção básica? Integração do PSF à rede de serviços de saúde. Saúde Debate 2003; 27(65): 278-89.

4. Organización Panamericana de la Salud. Sistema de Estrategia Salud basados en la atención primaria de salud: estrategias para el desarrollo de los equipos de APS. Washington: OPAS; 2005.

5. Ministério da Saúde (BR). Secretaria de Atenção à Saúde. Departamento de Atenção Básica. Política Nacional de Atenção Básica. Brasília: Ministério da Saúde; 2006.

6. Ministério da Saúde (BR). Secretaria de Atenção à Saúde. Saúde da família: uma estratégia para a reorientação do modelo assistencial. Brasília: Ministério da Saúde; 1997.

7. Paim IS. A reforma sanitária e os modelos assistenciais. In: RouQuayrol MZ, Almeida N, organizadores. Epidemiologia e saúde. $5^{\text {a }}$ ed. Rio de Janeiro: Medsi; 1999.

8. Teixeira CF. A mudança do modelo de atenção à saúde no SUS. Saúde Debate 2003; 27(65): 257-77.

9. Perrenoud P. Construir as competências desde a escola. Porto Alegre: Artmed; 1999.

10. Deluiz N. Qualificação, competências e certificação: visão do mundo do trabalho. Formação 200 I; I (3): 5-15.
1 1. Witti RR, Almeida MCPA. Competências profissionais de saúde no referencial das funções essenciais de saúde pública: contribuição para a construção de projetos pedagógicos na enfermagem. Rev Bras Enferm 2003; 56(4): 433-8.

12. Organización Panamericana de la Salud . Funciones esenciales de salud publica: una perspectiva desde las prácticas sociales: marco de referencia para una discusión regional. Washington: OPAS; 1988.

13. Minayo MCS. O desafio do conhecimento. $9^{\mathrm{a}}$ ed. São Paulo: Hucitec; 2006.

14. Ribeiro EM. As várias abordagens de família do Programa Saúde da Família (PSF). Rev Latinoam Enfermagem 2004; 12(4): 658-64.

15. Franco T, Merhy EE. PSF: contradições e novos desafios. [citado em 13 set 2006]. Disponível em: http://www.datasus.gov.br/ cns/temas/tribuna.htm

16. Travassos C, Martins M. Uma revisão sobre os conceitos de acesso e utilização dos serviços de saúde. Cad Saúde Pública 2004; 20(supl 2):5190-8.

17. Hartz ZMA, Contandriopoulos AP. Integralidade da atenção e integração dos serviços de saúde: desafios para avaliar a implantação de um "sistema sem muros". Cad Saúde Pública 2004; 20(supl 2):2531-6.

18. Crevelim MA. A participação da comunidade na equipe de saúde da família: como estabelecer um projeto comum entre trabalhadores e usuários? Ciênc Saúde Coletiva 2005; 10(2): 323-31. 